\title{
Analysis of laboratories electrical energy consumption by visualization for saving electrical energy
}

\author{
Ichinari Sato *, Diptarama *, Kaizaburo Chubachi *, \\ Ryo Yoshinaka *, Ayumi Shinohara *
}

\begin{abstract}
Saving electrical energy is an important task because the energy consumption is increasing but the resources are limited. Analyzing the electrical energy usage pattern of an institute is important to find a method to save electricity because the method to save electricity can be different depending on the usage pattern. Visualization is a good method to analyze the usage pattern and to present the analysis result to the public. In this paper, we analyze and visualize the electric energy consumption of all laboratories in a building. We also develop a system that automatically processes the electrical energy consumption and visualizes it in real time.
\end{abstract}

Keywords: energy saving, data visualization, energy consumption, heatmap

\section{Introduction}

We cannot imagine our daily life without electric energy in these days. Electric energy is indispensable in modern life in every aspect including private, industrial, and academic activities. Fossil fuels have been used as one of our main energy resources [1] for a long time. However, these resources are limited and the energy consumption is keep increasing. In Japan, after the Fukushima Daiichi nuclear disaster, some nuclear power plants have been stopped and electricity costs went up greatly. Therefore, energy saving becomes a more important issue for daily life including academic research activities, which this paper focuses on with respect to electricity consumption.

A comfortable research environment requires a large amount of electric energy consumption in general. We can reduce the electrical energy consumption by simply limiting the usage of lighting, air conditioners, or research equipment. However, it can reduce the efficiency of research activities. We need to find effective methods for electrical energy saving that can be applied without reducing the efficiency of research. Based on these motivations, we are executing a project to save electric power as academic researchers.

Efficient ways to save electricity in living space is studied in [2,3]. They proposed systems called smart building, smart office, and smart home to save electric energy consumption. Those systems get electric energy consumption in real time and to send feedback by sensor and wireless network like IoT. However, these smart buildings do not tell us a specific way for saving energy. We should find out the way in feedback information by ourselves.

\footnotetext{
${ }^{*}$ Tohoku University, Sendai, Japan
} 
In order to find a good method to save the electric energy consumption, we need to understand energy consumption patterns. To visualize them is a good method to understand energy consumption patterns. Various types of research on visualization of electric energy consumption have been shown, such as "time-pie visualization" [4] [5], "long-term polyline chart" and "one-day polyline chart" [6]. Visualization has two benefits. First, visualization does not need to use a complex technique to analyze data. Second, common people can understand the result of visualization easily.

Tohoku University already has a monitoring system for energy consumption [7] and we can browse the output through a web browser at any time. Unfortunately, the system only reports the total amount of energy consumption of all campuses in our university. Furthermore, the data is updated only once an hour. Therefore, people who want to save electricity can get only little information about how much power they consume at the current time. We cannot figure out a good method to save the electric energy by using this data only.

A more fine-grained and more interactive system is required to encourage the people to save electricity. We are developing a system that visualizes electrical energy consumption of all rooms in a building. We use heat maps to show electrical energy consumption, so that we can see the pattern of the consumption clearly and easily. By analyzing the result, we will find how to effectively save electrical energy. In this paper, we use data collected for nine months from November 2015 to July 2016.

\section{Meter System}

We use a system that records electric energy, water, and gas consumption. The system consists of meters that measure the consumption of each resource type and a server that records and manages the measured value from each meter. The system records each meter value minutely and produces a CSV file once an hour, which shows accumulated values for each meter. We analyzed the electrical energy consumption from these CSV files.

Concerning electric energy, accumulated values of power consumption per minute are recorded in units of $\mathrm{kWh}$ for each power switchboard. We categorized these switchboards into four types depending on the purpose of the room that each switchboard is placed; (1) shared space such as lecture rooms and corridors, (2) shared server room where several computer servers demanding high electric power are gathered, (3) shared laboratory (experimental room) where large-size experimental equipment is installed, and (4) individual laboratories, where laboratory members spend the most time. We then analyzed the electrical energy consumption differently by each category.

\section{Visualization}

A standard approach for data analysis is to perform exploratory analysis and explanatory analysis alternately. In the exploratory analysis we build a hypothesis from data, and in the explanatory analysis we find a way for saving electricity. In this research, we first get several hypotheses from monthly summaries of energy consumption. Then we visualize data to get more detail, and verify these hypotheses. 


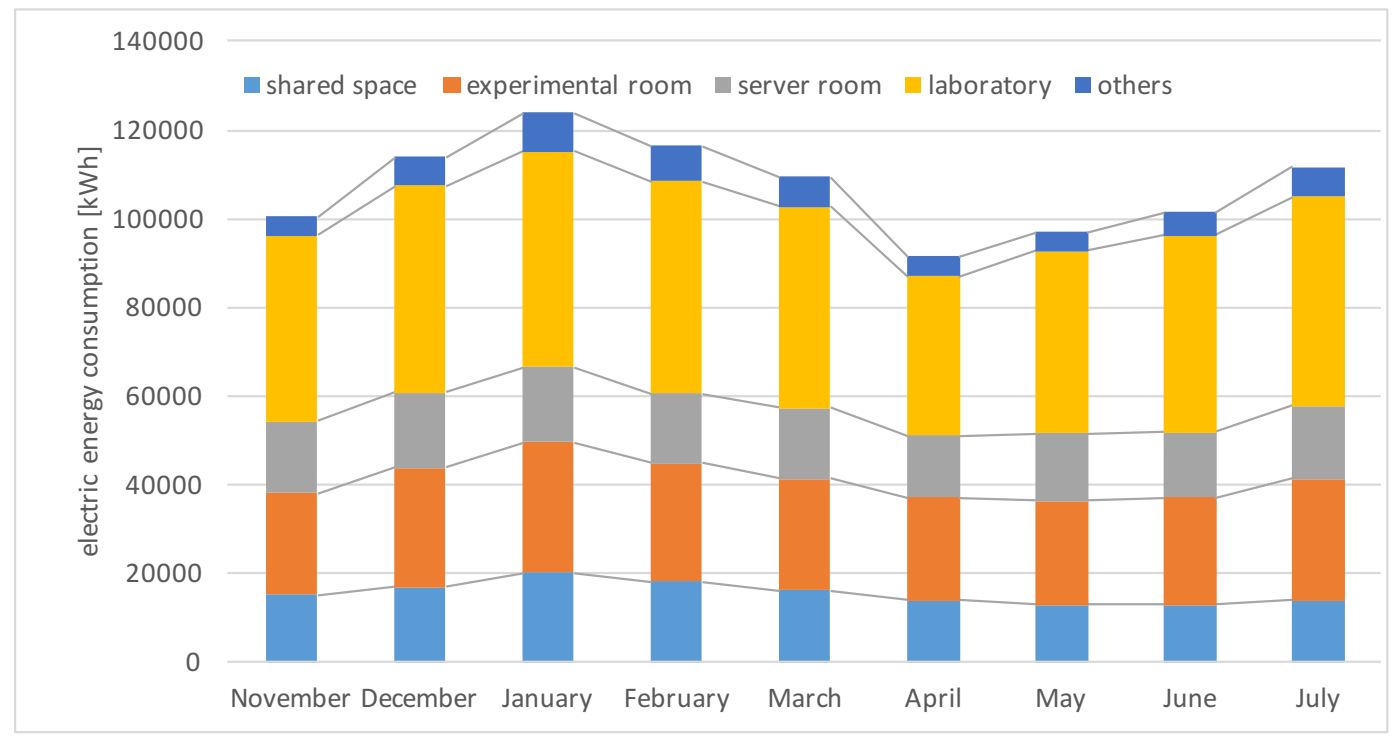

Figure 1: Monthly energy consumption, November 2015 - July 2016.

\subsection{Switchboard overview}

We summarized the monthly electric consumption data into a simple stacked chart in Fig. 1. Each color in the stacked chart corresponds to a type of switchboard. The chart shows a trend that electric energy consumption has increased month by month from November to January and from April to July. We also can see that electrical energy consumption has decreased from January to February and from March to April. The main factors of change in the chart are due to the changes in electrical energy consumption in experimental rooms and laboratories.

The result implies that the issue to be dealt with is (1) how to cut down the baseline consumption, and (2) suppress monthly increase, for all types of rooms, in order to decrease the total consumption. For that purpose, we have to find why consumption increased. We consider that the following may be major factors. (1) baseline consumption: standby power of various electric appliances. Air ventilation system might be another major factor. (2) monthly increase: air conditioner, especially heater in winter season. In the rest of this paper, we focus on the individual laboratories and investigate these factors in a more detailed analysis.

\subsection{Daily data visualization}

The baseline of electrical energy consumption can be obtained from the periods of time when nobody is working in the room, typically either midnight or holidays. First, we compare electrical energy consumption on weekdays and weekends by plotting the average consumption of all individual laboratories into box plots and violin plots as shown on Fig. 2. The box plot is represented by a white dot and black line in the middle of violin plot curve of each day. A white dot indicates the median in each distribution of energy consumption, and box edges indicate lower and upper quartile. The box plot shows us the exact values of these 3 indicators for the daily energy consumption, but it does not show the distribution shape of the consumption. Therefore, we see the density and the peaks of the distribution by using the violin plot. 


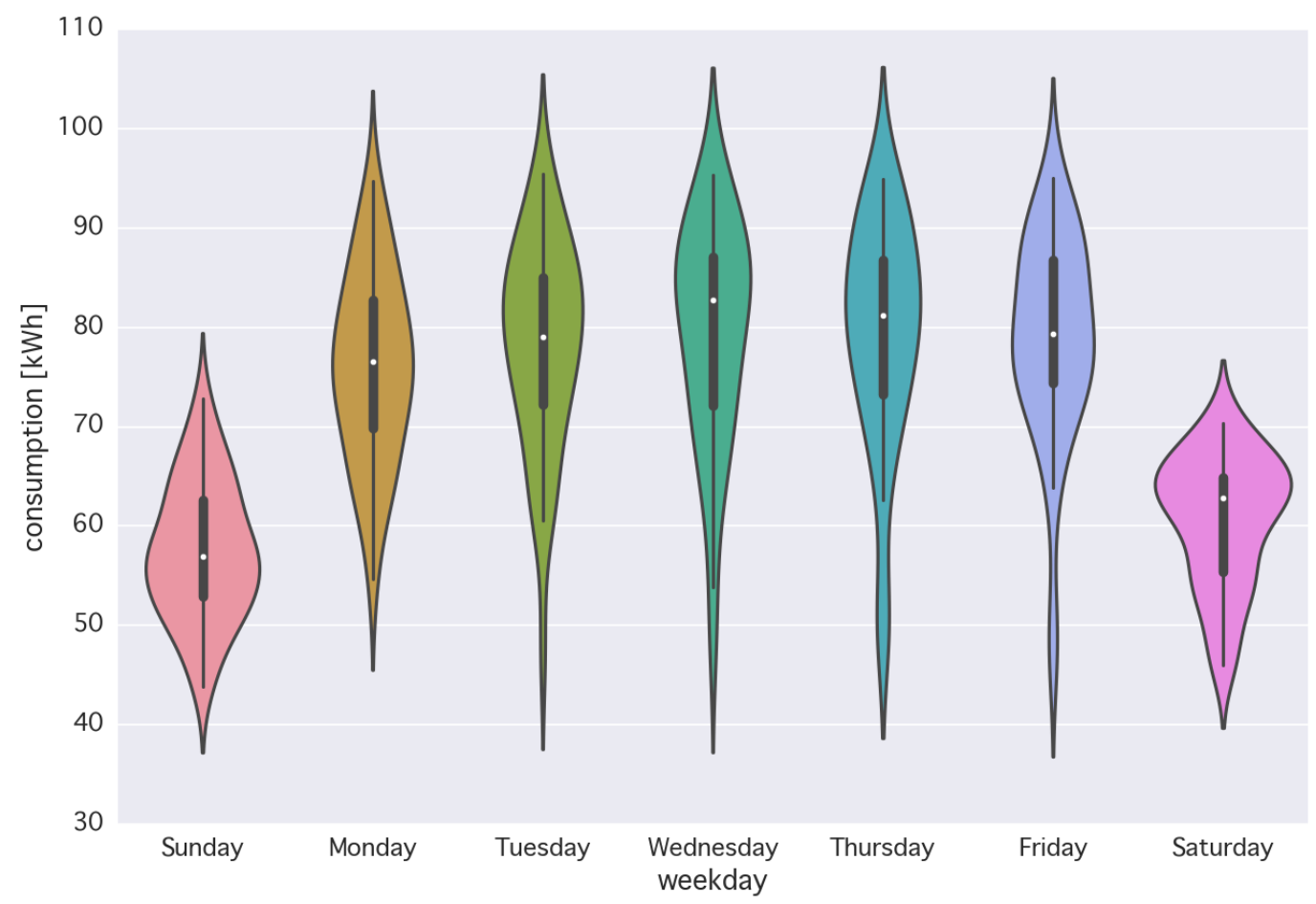

Figure 2: Average energy consumption of all individual laboratories for every day of the week.

We see that among weekdays, energy consumption is the smallest on Monday because Monday is often a national holiday due to the Substitute holiday and the Happy Monday System in Japan. In Japan, when a national holiday is fall on Sunday, the next Monday will become an alternative of Sunday, when people do not work usually. Moreover, many holidays are defined to be a Monday of a month. Those systems extends many weekends up to Monday. Although both Saturday and Sunday are weekend days, their distributions of energy consumption are different; the median and peak in Saturday are higher than those on Sunday.

We need more detailed analysis to understand the reason for the difference. Fig. 3 shows a heat map of the daily consumption by color strength, from the darkest $(95.4 \mathrm{kWh})$ to the lightest ( $43.8 \mathrm{kWh}$ ), aligned according to the day of week from the leftmost (Sunday) to the rightmost (Saturday).

The figure shows two trends; (1) energy consumption is low on national holidays, not only Saturday and Sunday, and (2) energy consumption in February is higher than that in November. We explain about this later.

The consumption in all national holidays is lower than the days before and after the holidays. Moreover, it is clear that the consumption are lower on consecutive holidays such as New Year's holidays and early in May. In spite of it, the day of lowest energy consumption is April 10th, Sunday. The energy consumption in the weeks around this day is also lower than other weeks. The first semester in each grade starts from April in Japan. Many students take guidances of some classes including classes that they would not take. We consider that students belonging to a laboratory tend to be absent from their laboratory to select classes in April.

The energy consumption on weekdays from December to February is higher than that in November. One of the major factors for this could be that many students spend more 


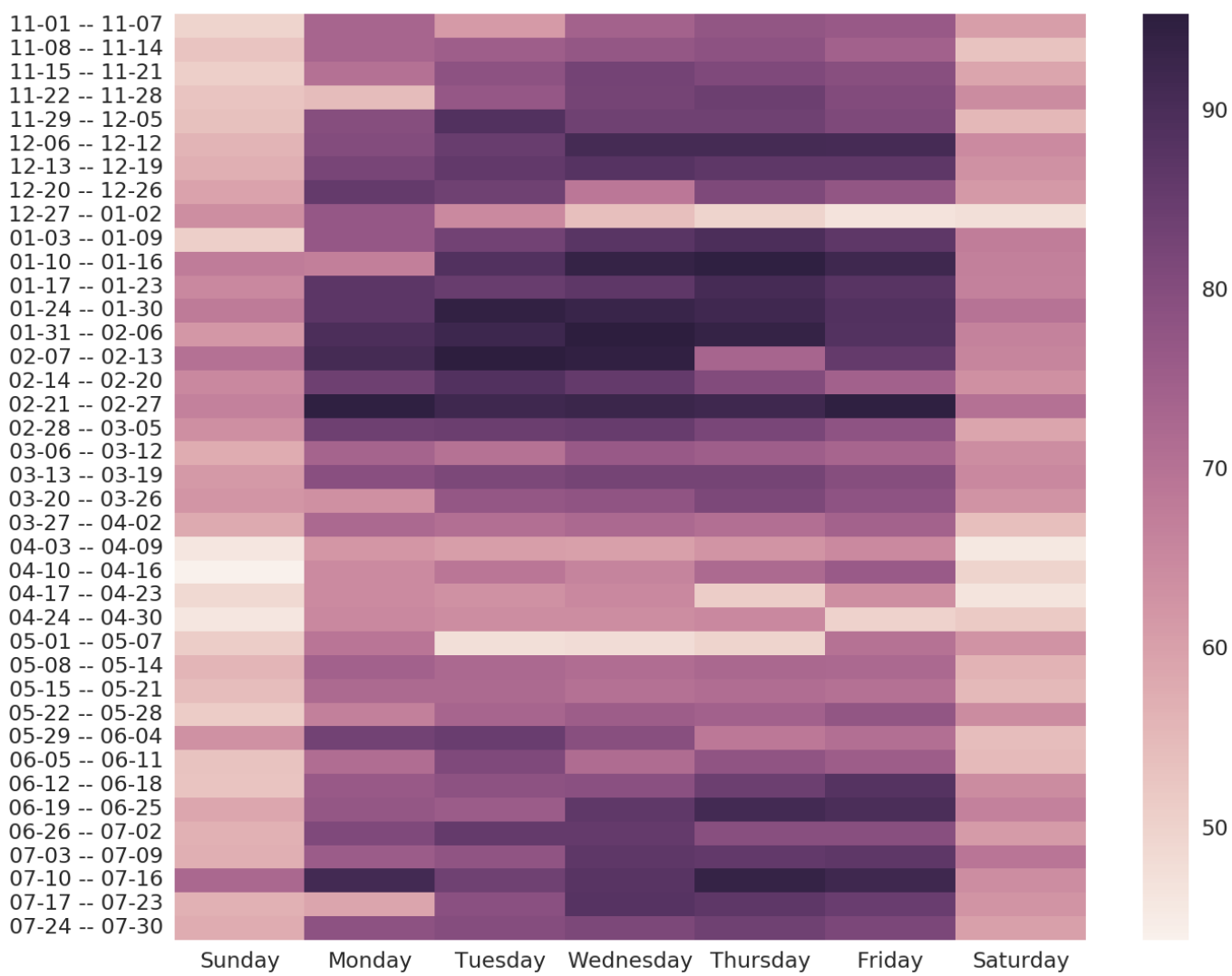

Figure 3: Daily energy consumption on average of all individual laboratories, aligned according to the day of the week. The dates are shown by $\mathrm{mm} / \mathrm{dd}$. Lighter cells in weekdays are holidays; November 3rd, November 23rd, December 23rd, January 1st, January 11th, February 11th, March 21st, April 29th, May 3rd, May 4th, May 5th, and July 18th.

time in their laboratories for the preparation of doctoral, master, and bachelor examination. For example, the preliminary examination term for master thesis was from January 13th to 16th and the final examination term was from February 12th to 16th. On the other hand, the preliminary examination term for doctoral dissertation was from November 26th to 27th and the final examination term was from January 21st to 22nd. Almost all laboratories carried out reviews for bachelor thesis from later in February to early in March. Since the examination of bachelor thesis depends on the policy of each laboratory, the examination schedule for bachelor student was not fixed.

This hypothesis is supported by the fact that the energy consumption is rather high at February 11th even though the day is a national holiday. These observations lead us to consider the effect of seasonal academic activities, not only the seasonal climatic effect such as an air conditioner (heater and cooler) that are already pointed out in the previous section.

\subsection{Hourly data visualization}

By the daily heat map visualization (Fig. 3) in the previous section, we confirmed that the energy consumption has a pattern depending on the day of the week and that it is lower on holidays. We now turn our attention to the daily fluctuation. 
Two types of switchboards are used in our building, one for standard plug sockets, and the other for air conditioners only. We note that plug sockets manage also room lights in laboratories. We will explain them separately.

For each switchboard for plug sockets, we created a heat map of the hourly consumption by color strength, aligned according to the time of day. In Fig. 4, we plotted some of the switchboards. We observed that all these heat maps can be classified into three categories, depending on the patterns. Plug socket A, B, and C in Fig. 4 are representatives of these three categories, respectively.

Category 1 (represented by plug socket A): A typical heat map of a switchboard in an individual laboratory, where students spend most of their time. 22 out of 55 switchboards are classified in this category. We note that the heat map of energy consumption on average of all individual laboratories has the same pattern in this category. The pattern clearly reflects their lifestyle in the laboratory; they usually come to the room in daytime on weekdays and sometimes stay until midnight or to the next morning. We think that this is one of the major factors why the mean energy consumption on Saturday is higher than that on Sunday in Fig. 2; some students stay until Saturday morning, but few stay until Sunday morning.

Category 2 (represented by plug socket B): The energy consumption does not change very much over time. 17 out of 55 switchboards are classified in this category. This is typical for the switchboards in server rooms.

Category 3 (represented by plug socket C): The energy consumption changes sharply and focuses on specific time periods. 16 out of 55 switchboards are classified in this category. This is typical for the switchboards in experimental rooms.

As we have seen, energy consumption patterns are quite different depending on the purpose of a room, even if the rooms are located in the same building. Therefore, careful analysis is indispensable to cut down the energy consumption effectively.

Each pattern is clearly different from the others, so it would be easy to guess the usage of a room by only observing energy consumption of the switchboards in the room. Although saving electric energy is very important, saving should not disturb research activities. In this sense, there is little hope to save energy consumption of experimental rooms such as switchboards B and C in Fig. 4. On the other hand, if we succeed in reducing the electric energy consumption of a room in Category 1 and apply it to similar rooms, we can save a significant amount of energy consumption in total.

Let us focus on Category 1 in more detail, and consider the relation between the energy consumption and the number of persons who usually use the room. Other than plug socket $\mathrm{A}$, we selected two plug socket switchboards, shown by plug socket $\mathrm{D}$ and $\mathrm{E}$, in Fig. 4. The numbers of persons who use the room with plug socket A, D, and E are 45, 25, and 13, respectively. We can conclude that on the socket switchboards from Category 1 , energy consumption scale is proportional to the number of laboratory members. To check the hypothesis we plotted daily energy consumption of each laboratory and the number of members belonging to the laboratory to scatter diagram (Fig. 5). Unfortunately, we did not find out correlation from this scatter diagram. Note that we counted the number of members by visiting websites of all laboratories in December 2015, and the number was necessarily equal to the actual number of persons who usually use the room.

We now turn our attention to the switchboards for air conditioners. Similarly to the switchboards for plug sockets, the heat map of energy consumptions can be classified into at least two categories. Air conditioner A from Category 1 is in an individual laboratory room, and the pattern is closely related to the pattern for the switchboards for plug sockets. Air conditioner B from Category 2 is in an experimental room, for controlling the tempera- 


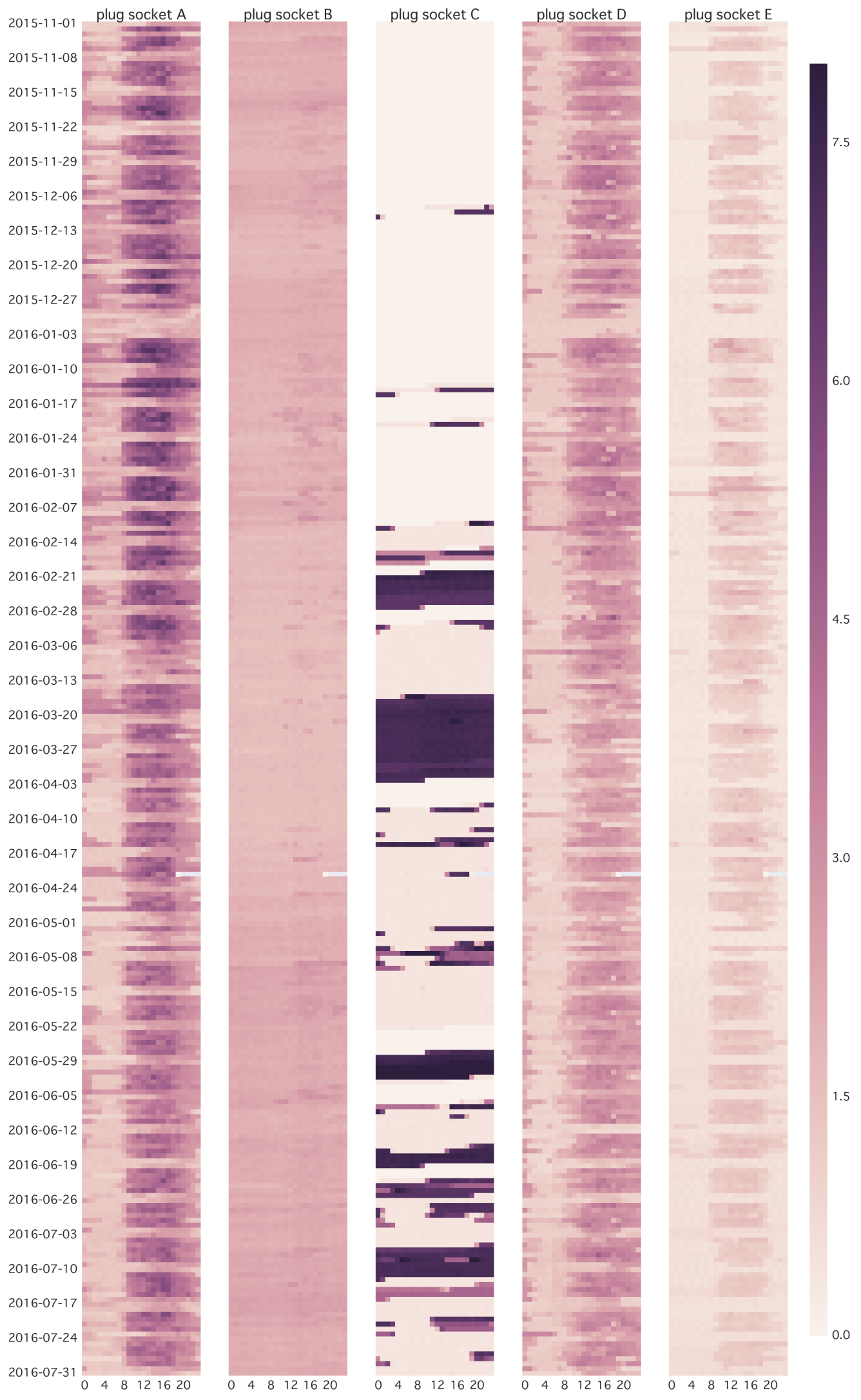

Figure 4: Hourly energy consumption measured on switchboards in individual laboratories. The dates are shown by yyyy-mm-dd. Switchboards for plug sockets. The color scale range is between $0 \mathrm{kWh}$ and $8 \mathrm{kWh}$. 


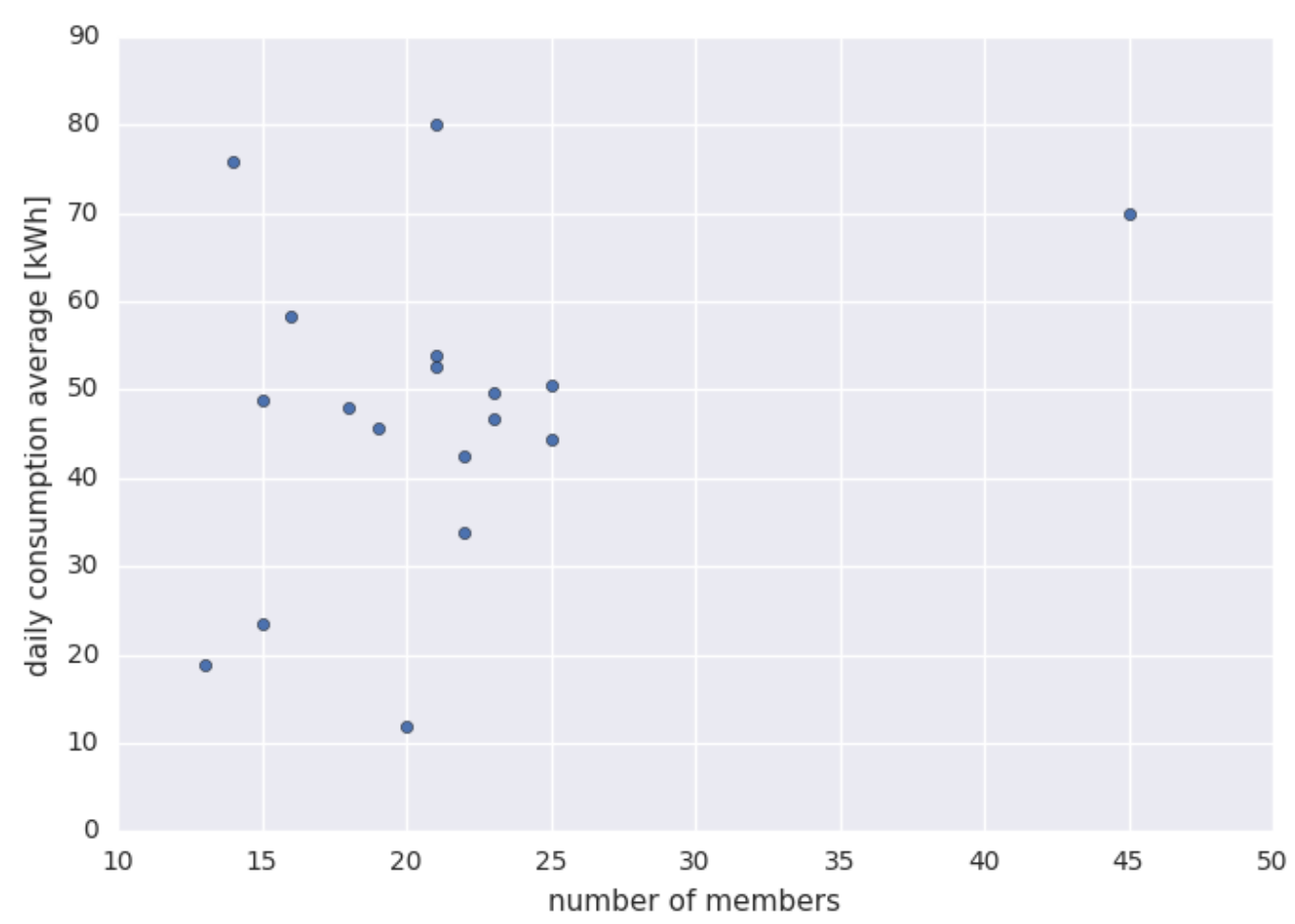

Figure 5: A scatter diagram consists of daily electrical energy consumption from switchboards in Category 1 and the numbers of members belonging to the laboratory.

ture and humidity. The room was used since the end of December, after some preparations in November. These activities can be recognized from the heat map. Fig. 6 shows representatives from these categories, respectively.

In Section 3.1, we built a hypothesis that the air conditioners would be a major factor of increasing energy consumption from November to January. The hypothesis is consistent with the heat map of air conditioner A in Fig. 6, where energy consumption gradually increased since the middle of November 2015. The hypothesis is also supported by the fact that the temperature fell during those days. Restricting air conditioner usage is not really effective for saving energy because consumption values by air conditioner are small.

\section{Idea for saving energy}

In this section, we suggest two ideas for saving electric energy consumption, based on further analysis of electric energy consumption of category 1 plug socket. The first idea is from daily electric energy consumption pattern changes of some laboratories. The second idea is from seasonal pattern of electrical energy consumption.

\subsection{Time variation}

We further analyze hourly electric energy consumption pattern of category 1 to find an idea to save the electric energy. Fig. 7 shows hourly electric energy consumption of Category 1 

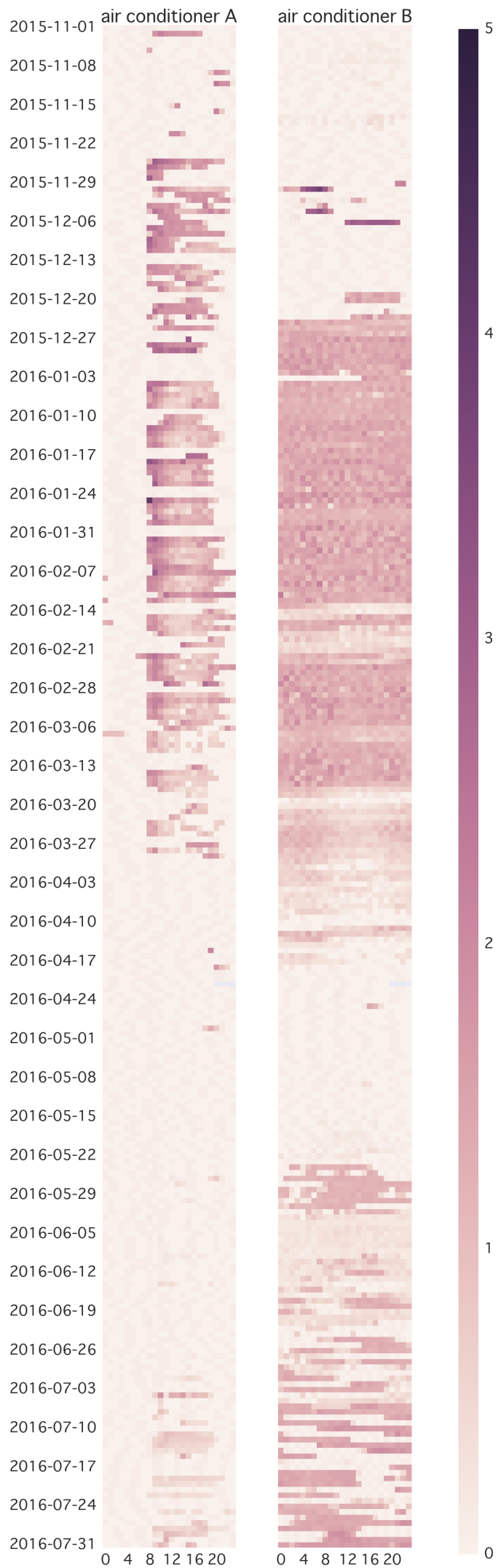

Figure 6: Hourly energy consumption of air conditioners in individual laboratories, $0-5 \mathrm{kWh}$. The dates are shown by yyyy-mm-dd. 


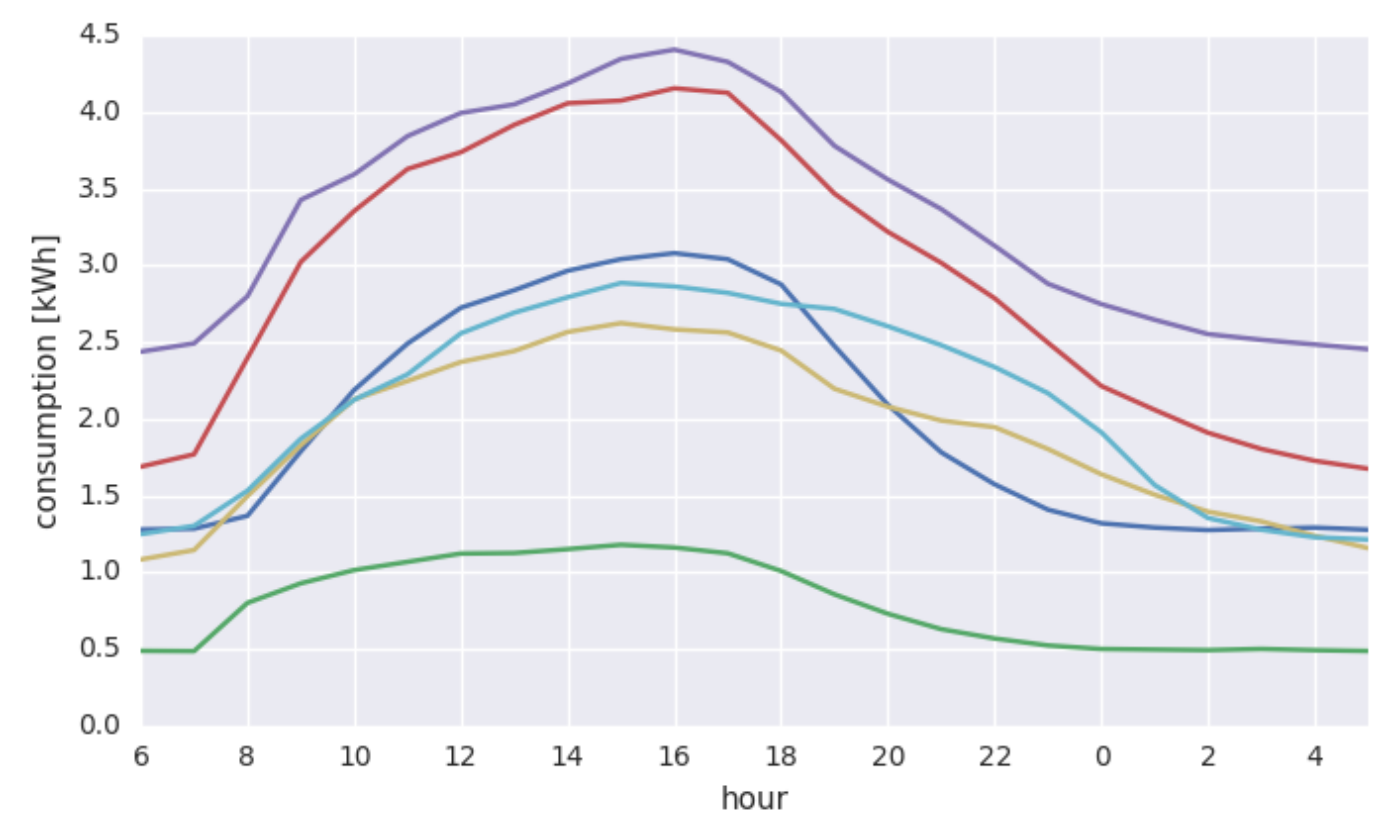

Figure 7: Hourly electric energy consumption of Category 1 of several laboratories.

of several laboratories. As we can see from this figure, in almost all laboratories the consumption increases sharply from 7 a.m. to 8 a.m. But there is no such sharp fluctuation in decreasing after passing the peak of the consumption. It is because that many people come at the same time around 8 a.m., while there is variation in time when people leave the laboratory. This variation is less important for electricity consumption of devices used by an individual such as personal computers, but it is important for electricity consumption of devices shared by some people such as lighting because these devices spend electricity until all the people leave the laboratory. Therefore, electricity consumption can be saved if all the members in a laboratory come and go back at the same time. It will promote people to research more efficiently in shorter time, instead of using too much time. For the people who want stay longer, they should study in a place where they do not use shared devices too much.

In order to estimate the effect of this approach, we pick up two laboratories A and B and compare them in Fig. 8. After the peak of consumption, the energy consumption by laboratory A is more sharply decreased than laboratory B. Comparing these two laboratories, although the peak value in laboratory $\mathrm{A}$ is higher than that in laboratory $\mathrm{B}$, the total value in laboratory A is $17.4 \mathrm{kWh}$ whereas $19.1 \mathrm{kWh}$ in laboratory B, so laboratory B spends electricity $2 \mathrm{kWh}$ more per day. We think that laboratory B can save some electrical energy by this approach. We also consider that the consumption can be further reduced even in the laboratory A, so that more electricity can be saved by this idea.

\subsection{Seasonality in category 1}

In Fig. 9, the average of daily energy consumption of laboratories measured on switchboards in Category 1 is shown by blue line. We also plotted the simple moving average of past 7 days by red line to exclude weekly cycles. As we have seen in Section 3.2, seasonal 


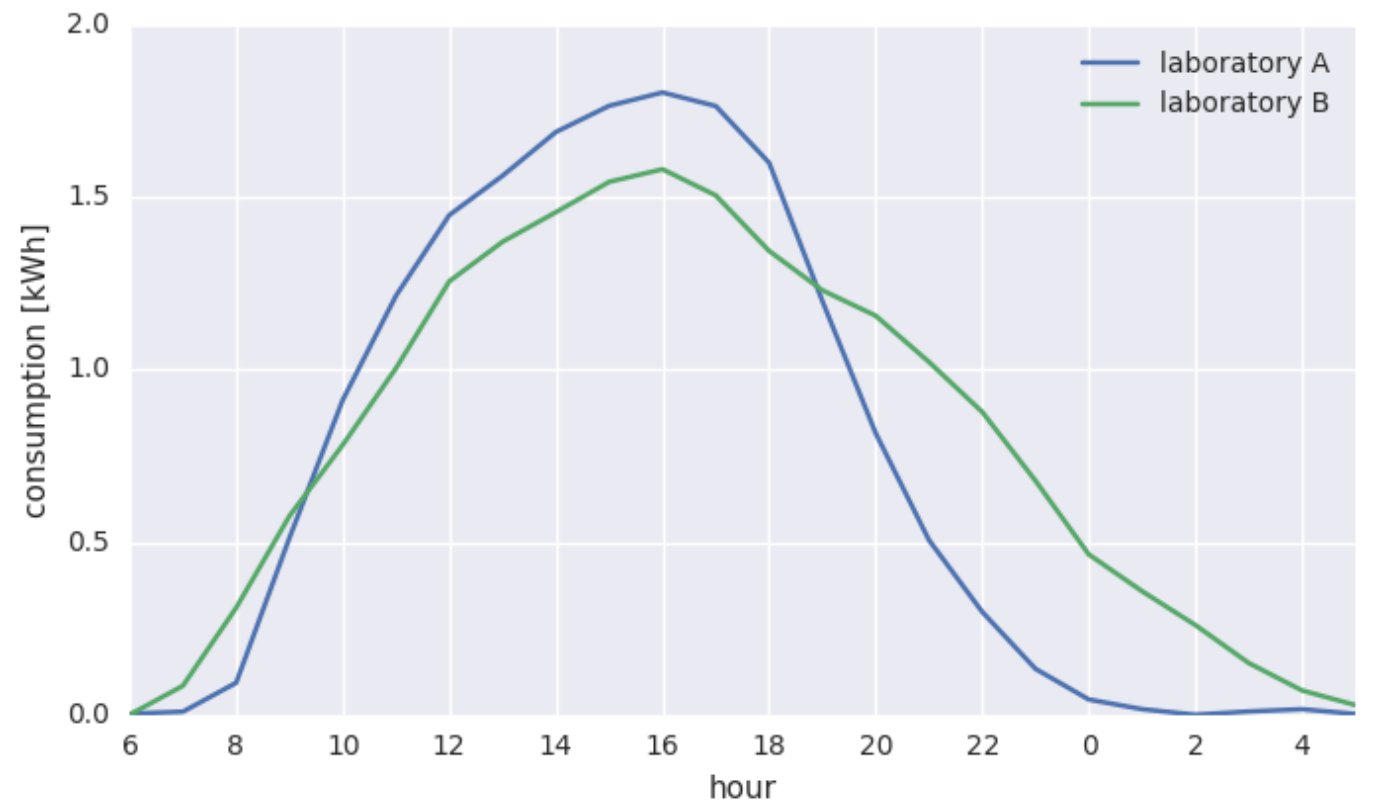

Figure 8: Hourly electric energy consumption of Category 1 of laboratories A and B. To remove the difference of the baseline consumption of these laboratories, we show the consumption as the difference from minimum consumption of each laboratory.

academic activities affect the consumption. Although this chart omitted some switchboards in Categories 2 and 3, we can see in Fig. 9 that the energy consumption is increasing in winter and decreasing in spring. The reason is that in winter season most people come to the laboratories for preparing the graduation examination and new laboratory members come in this season. On the other hand, the period from March to May is a season when there are less people in the laboratories because some students have graduated and some left for another city to find a job. Therefore, considering laboratory members' activities which can be indirectly observed in Fig. 9, we can conclude that winter is the most effective season to save electricity consumption whereas April is less.

\section{Visualization website}

As we have shown in the previous sections, the electric energy consumption in individual laboratory rooms is directly affected by research activities performed there. We are developing a web system that interactively and continuously visualizes the energy consumption in each laboratory for a selected time scale. The website provides two kinds of views of the data automatically extracted from the CSV files.

On the main page (Fig. 10), a user can get a general view of the current energy usage at all laboratories as a heat map. Moreover, the user can specify any past time to replay an animation of temporal data and compare with other rooms.

By clicking any room shown on the home page, another view (Fig. 11) appears. It displays a time series of the consumption in the room during a specified time period. The time unit scale can be also selected from either 5 minutes, 1 hour, 1 day, 1 week, 1 month, 


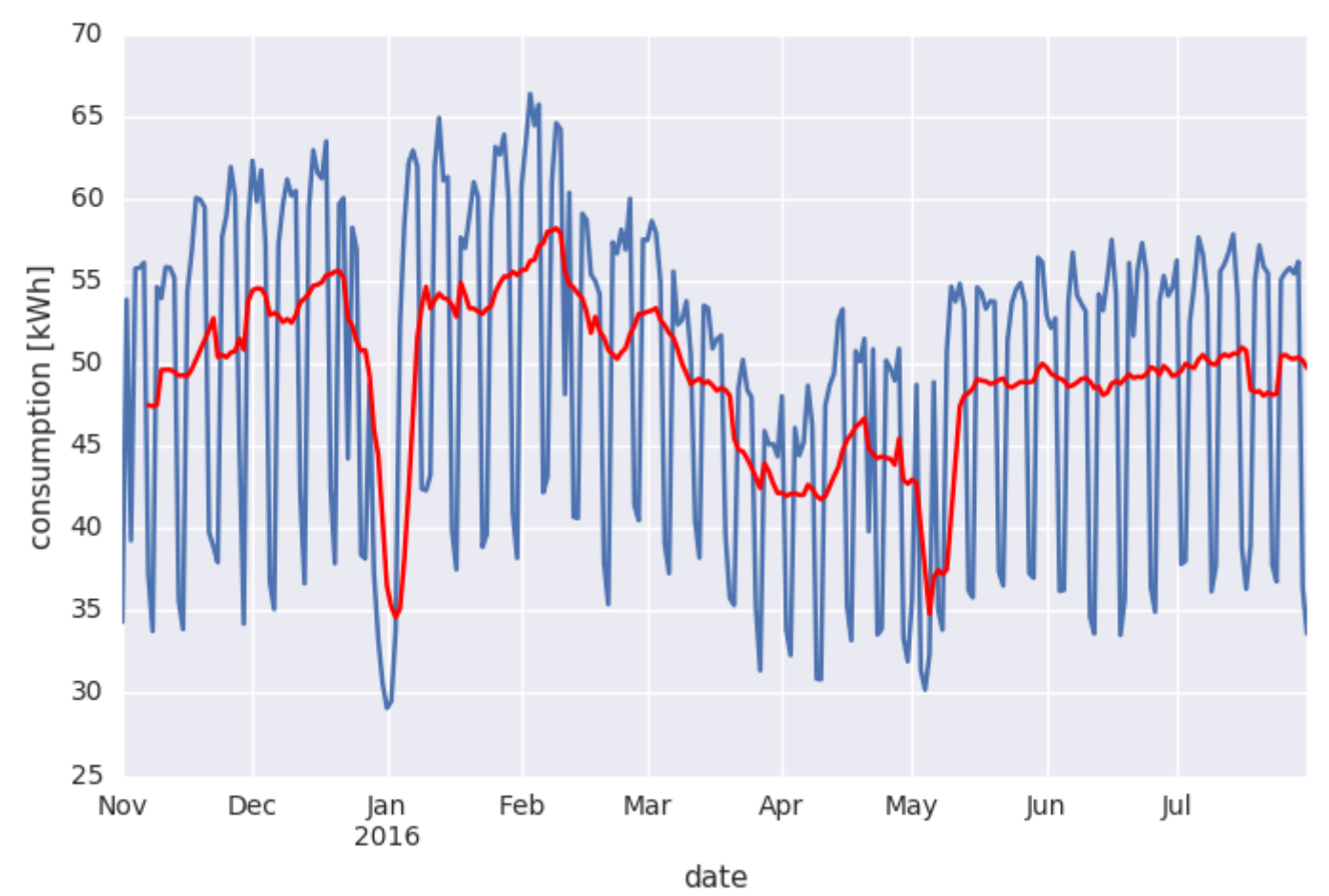

Figure 9: Daily electric energy consumption of Category 1. The blue line shows daily average values and red line shows the simple moving average of past 7 days. The red line falls down sharply in consecutive holidays such as New Year's days and early in May. Moreover, there is a gentle decrease from March to April. We consider that this decrease is due to students' graduation and job hunting.

1 year, or all. Each color in the chart of Fig. 11 corresponds to a single switchboard, where one laboratory can have multiple switchboards.

\section{Conclusion}

As a result of our analysis in this paper, we found two points for saving energy:

(1) reducing usage of shared devices when less members are in a laboratory, and

(2) working out (1) in winter.

Through various cycles such as season, the day of the week, and time in the day, the peak power usage in our building may occur during a day in winter. In fact, we confirmed that the peak zone for our building coincides with the peak zone for the whole campus in Tohoku University (see [8], page 13). For the moment, we have collected the energy consumption data only for nine months. If the peak zone and distributions of the energy consumption continue to match for a year or several years, we can regard that the knowledge in this building would be applicable for the whole campus. 


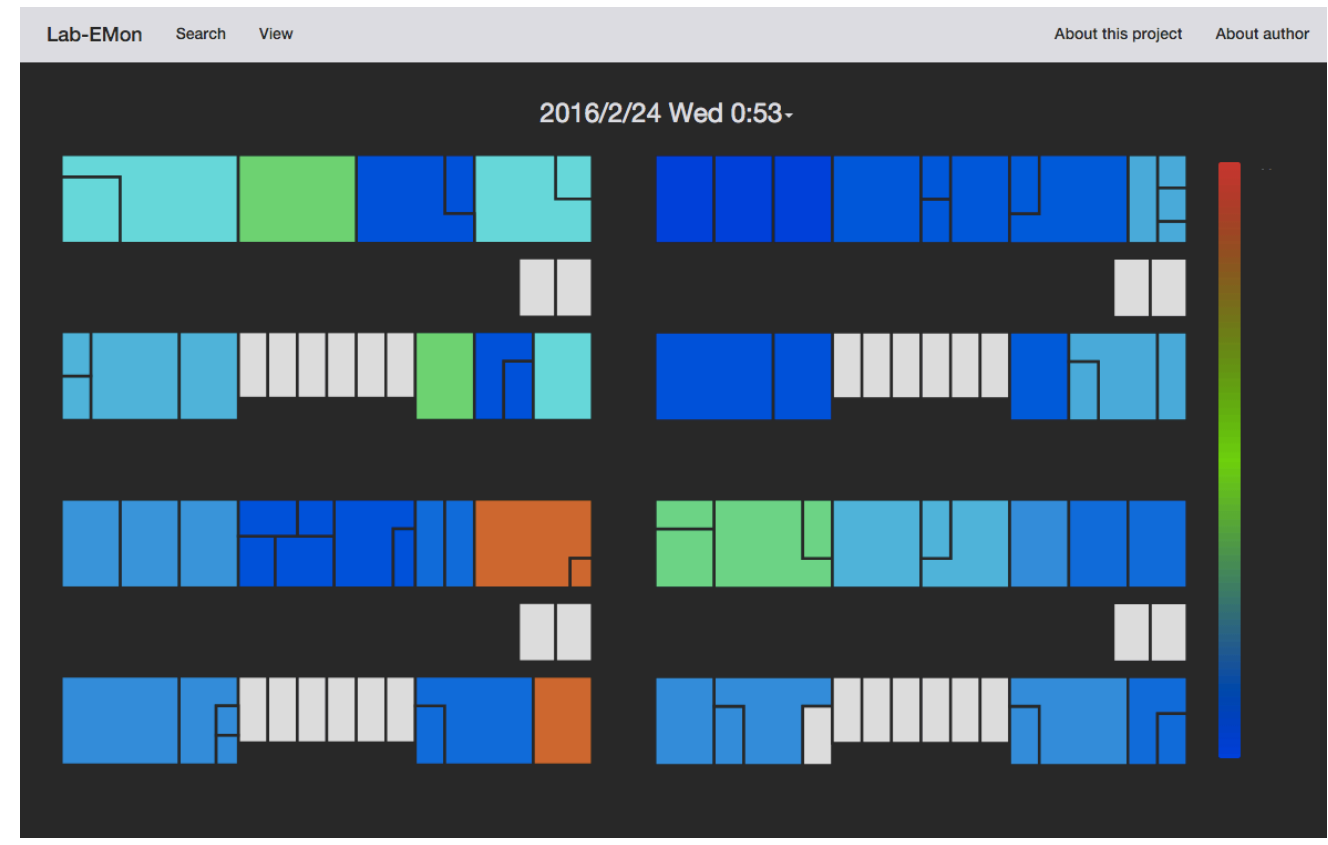

Figure 10: The home page of the website gives an overview of the energy consumption of all rooms.

\section{References}

[1] International Energy Agency. Electricity information, 2015 edition, excerpt. http://www.iea.org/publications/freepublications/publication/ Electricitytrends.pdf.

[2] C. Wei and Y. Li. Design of energy consumption monitoring and energy-saving management system of intelligent building based on the internet of things. In Electronics, Communications and Control (ICECC), 2011 International Conference on, pages 3650-3652, 2011.

[3] B. Asare-Bediako, P. F. Ribeiro, and W. L. Kling. Integrated energy optimization with smart home energy management systems. In $20123 \mathrm{rd}$ IEEE PES Innovative Smart Grid Technologies Europe (ISGT Europe), pages 1-8, 2012.

[4] M. Masoodian, B. Endrass, R. Buhling, P. Ermolin, and E. Andre. Time-pie visualization: Providing contextual information for energy consumption data. In Information Visualisation 2013, 17th International Conference, pages 102-107, 2013.

[5] M. Masoodian, B. Lugrin, R. Buhling, and E. Andre. Visualization support for comparing energy consumption data. In Information Visualisation 2015, 19th International Conference, pages 28-34, 2015.

[6] T. Itoh, M. Kawano, S. Kutsuna, and T. Watanabe. A visualization tool for building energy management system. In Information Visualisation 2015, 19th International Conference, pages 15-20, 2015.

[7] Tohoku university power monitoring system. http://denryoku-monitor. tohoku. ac. jp. 


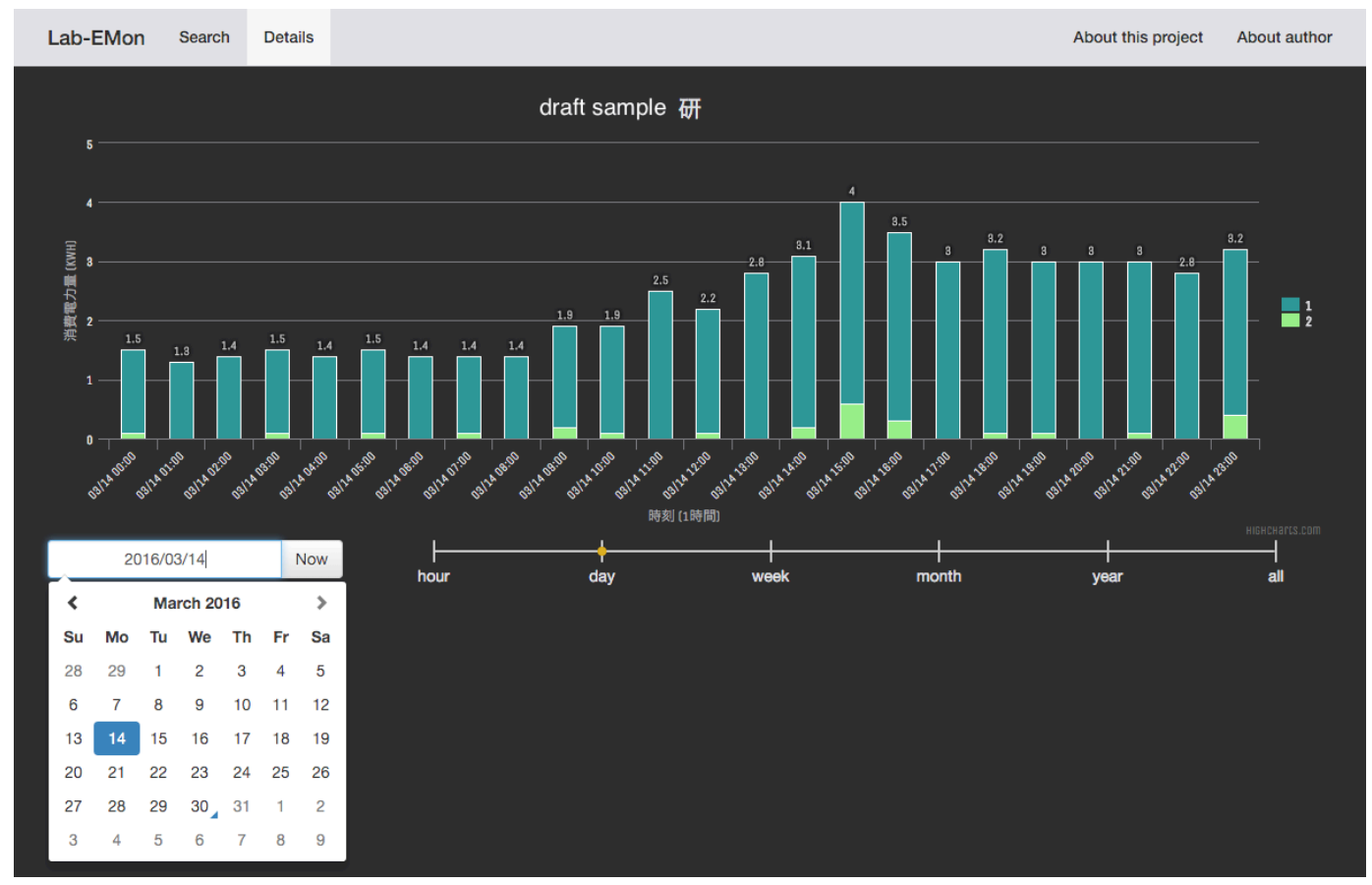

Figure 11: Another view presents a time series of the energy consumption of the specified room. The time scale and period can be changed.

[8] Tohoku university environmental management report 2015. http://www. bureau . tohoku.ac.jp/anzen/env_mane_pro_office/file/04-01-19.pdf. 